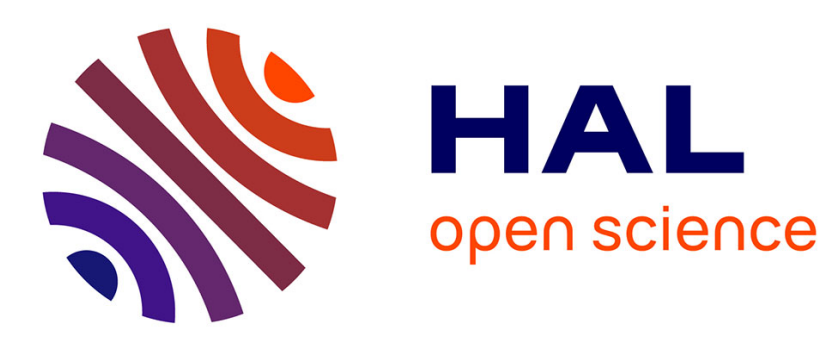

\title{
Symbolic environment representation by means of frescoes in mobile robotics
}

\author{
Gilbert Pradel, Philippe Hoppenot
}

\section{To cite this version:}

Gilbert Pradel, Philippe Hoppenot. Symbolic environment representation by means of frescoes in mobile robotics. Robotica, 2005, 23, pp.527-537. hal-00341324

\section{HAL Id: hal-00341324 \\ https://hal.science/hal-00341324}

Submitted on 17 Apr 2014

HAL is a multi-disciplinary open access archive for the deposit and dissemination of scientific research documents, whether they are published or not. The documents may come from teaching and research institutions in France or abroad, or from public or private research centers.
L'archive ouverte pluridisciplinaire HAL, est destinée au dépôt et à la diffusion de documents scientifiques de niveau recherche, publiés ou non, émanant des établissements d'enseignement et de recherche français ou étrangers, des laboratoires publics ou privés. 


\title{
Symbolic environment representation by means of frescoes in mobile robotics
}

\author{
Gilbert Pradel \\ Laboratoire IBISC \\ Université d'Evry Val d'Essonne, CNRS FRE 2873 \\ 40,rue du Pelvoux, CE 1455 Courcouronnes, 91020 Evry cedex. FRANCE \\ Email: gpradel@ibisc.univ-evry.fr \\ Philippe Hoppenot \\ Laboratoire IBISC \\ Université d'Evry Val d'Essonne, CNRS FRE 2873 \\ 40,rue du Pelvoux, CE 1455 Courcouronnes, 91020 Evry cedex. FRANCE \\ Email: hoppenot@ibisc.univ-evry.fr
}

\begin{abstract}
Autonomous mobile robot navigation systems are based on three principal kinds of techniques: map-based navigation, map-building-based navigation and mapless navigation. We propose a mapless method for trajectory description in unknown indoor environments. The method uses distance measurements from a 2D laser range finder, digitises the robot's visibility area, eliminates superfluous data and reorients their presentation with laws similar to those used in cellular automata. The landmarks are extracted and organised in a panoramic description called fresco. The frescoes which are validated by means of neighbourhood rules. The most informative frescoes are detected by means of two criteria and stored. The stored frescoes are considered as a human-like descritption of the robot's route and could be used by the robot to retrieve its route to its starting point.
\end{abstract}

\section{INTRODUCTION AND RELATED WORKS}

The main issue for mobile robots is their capacity to go from one point to another autonomously. It is based on three concepts: i) planning which computes a trajectory between the two points, ii) navigation which gives motion orders to the robot to follow the computed trajectory and iii) environment representation which allows the robot to know if it goes in the right direction. Works presented here are interested in point iii), trying to perform a qualitative description of a stuctured indoor environment. This description should be used by a middle-cost mobile robot that is sent in an apartment to pick up objects in order to bring them back to, for example, a physically handicapped person. We are aware that the robot cannot replace a person. The main interest that is seen in such system is its 
use while the immobilized person is alone. The environment is then considered as static, a priori unknown and therefore cannot be modelled in advance. Ideally, the mission, if based on the knowledge of room situation in the flat, could be given to the robot using orders very close to the natural language such as "Go to the kitchen, pick up the fork and bring it back to me" through an ad hoc interface. In fact, it is more realistic to think to a lower-levelled language based on a succession of topological markers. For example, a part of a mission could be: "move through the corridor, take the third opening on the left, enter the room" followed by a more precise description of the goal situation the robot has to find. In that case, the route and/or the place to go can be described both in global terms and by using landmarks. Whatever the skill of the language interpreter, the robot must have the ability to build a model restricted to very global and syntactical information. We choose to describe the surrounding environment by means of clues such as "Opening", "Closure", "End_of_Closure", "Angle_of_Closures", ... organised into ordered series called frescoes according to the data delivered by the sensor.

One immediately thinks to the memorisation of the environments that the robot chronologically covers during a journey. As the robot moves along the trajectory, it is also interesting to analyse how the frescoes are modified and transformed. In other words, what new information a fresco brings up in relation with the preceding ones? When all the uncertainties are solved the fresco - qualified as a relevant one - has to be stored in a long term memory. Another interesting problem to tackle is to use the pertinent frescoes stored in the long term memory to give the robot the ability to return to its starting point in a return journey.

In the field of Image Based Navigation systems, several great classes of systems can be identified from the literature. The first one uses conventional telemeters and vision to find and identify objects in the environment [1]. The second one is the class of the systems coupling more or less directly sensor data to motor control thanks to a supervised learning process. Among them neural networks systems used as classifiers are noticeable. These systems begin to classify the environment into global classes such as "corridor, corner, room, crossing ..." [2], [3] are often followed by a second processing unit that outputs a navigation command. In addition to restrictions related to the supervised learning, these classes give only a global description and are of least interest in cluttered and complex environments. The third class includes the systems which compare current sensor data and predefined models both at a low level (edges, planes ...) [4] and at a high level (door, room, object ...). These systems use mainly vision sensors (cameras) that provide a huge amount of data that must be reduced to be processed in real time. The elements extracted from the data are compared to reference models known a priori. The fourth class evoked here includes the systems trying to geometrically build environment models before deciding an optimised path plan [5].

Vision for mobile robot navigation did have specific development during the last twenty years.DeSouza [6] gives a complete survey of the different approaches. For indoor navigation, systems are classified in three groups: map-based navigation using predefined geometric and/or topological models, map-building-based navigation constructing by thmeselves geometric and/or topological models, and mapless navigation using only object recognition and actions associated to these objects [7].

In the field of shape understanding using sensor data, environment interpretation stresses the use of natural landmarks to ease the navigation and the pose estimation of a mobile robot. Among other works, one can pinpoint 
Simhon [8] which is interested in defining islands of reliability for exploration. He proposes strategies to couple navigation and sensing algorithms through hybrid topological metric maps.Oor [9] considers the problem of locating a robot in an initially unfamiliar environment from visual input while MacKenzie [10] involves a methodology to bind raw noisy sensor data to a map of object models and an abstract map made of discrete places of interest.

In the field of vision based homing, several implementations are presented in [11]. A method aiming at highlighting salient features, as for example landmarks, between these two views and deriving a decision is used in [12]. In these works, a homing system extracts landmarks from the view and allows a robot to move to home location using sequence target locations situated en route between its current location and home. Other works are biologically inspired. Judd [13] showed that ants store series of snapshots at different distances from their goal to use them for navigating during subsequent journeys. Judd and Collett experimented their theory with a mobile robot navigating through a corridor, homing successive target locations. Weber K. and Al. [14] propose an approach using the bearings of the features extracted of the panoramic view leading to a robust homing algorithm. This algorithm pairs two landmarks situated into two snapshots to derive the homing direction. The bearings pairing process uses a list of preferences similar to neighbourhood rules.

Symbolic processing methods are described in Tedder's works [15]. This formal approach is often called structural or syntactic description and recognition. The general method for perception and interpretation proposes to symbolically represent and manipulate data in a mapping process.Tedder solves the problem in modelling the 3D environment as symbolic data and in processing all data input on this symbolic level. The results of obstacle detection and avoidance experiments demonstrate that the robot can successfully navigate the obstacle course using symbolic processing control. These works use a laser range finder. A way for defining suitable landmarks from an enviuronment as the robot travels is a research problem pointed out in [16]. An automatic landmark selection algorithm chooses as landmarks any places where a trained anticipation model model makes poor detection. This model has been applied to the navigation of a mobile robot. An evaluation has been made according to how well landmarks align between different runs on the same route. These works show that the robot is able to navigate reliably using only odometric and landmark category information.

Simultaneous Localisation And Mapping (SLAM) methods have been introduced by Leonard and DurrantWhyte [17] and [18] showing that it is possible to associate the topological and geometric structure of an environment and its symbolic description. Kuipers [19] defined symbols as distinct places situated at equal distances from the nearby obstacles. Connections between these places link symbols and represent free path [20]. Figure 1 shows the Voronoii graph of an environment. In this figure, the labelled vertices represent the symbols while edges connecting the symbols are the path the robot can use. 


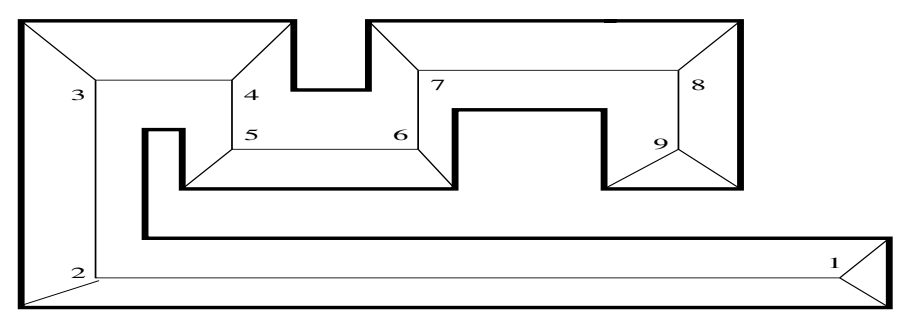

Fig. 1. Voronoii diagram whith numbers label symbols

The proposed work goes on the way proposed by Simhon [21] and Tedder [15]. According to these works, our contribution applies mainly on a method to extract clues of interest among raw distance data delivered by a 2D panoramic laser range finder installed on the robot. In addition to this qualitative approach, one must consider that the system will have to be embarked on a vehicle, which vibrates, runs at variable speeds on a non-uniform ground. This leads to constraints of speed, size, robustness, compactness and cost, implying various choices both at the design and at the development levels of the system. The methods used have been chosen as simple as possible to reduce the cost and the complexity of the processing. Nevertheless the method must be robust compared with the robot movements, the sensor accuracy and the variations of the complexity of the environment. Its originality stays in the digitisation process, the validation and the selection of the fresco for memorisation. The following points will be successively highlighted: 1) how the robot recognises and sets up a description of the surroundings, 11) how this description, the fresco, is validated, 111) how the clues are used to construct the fresco.

This paper is organised in 4 sections. Section II presents our approach in the digitised construction of the environment. Every steps from the overview of the perception system to the landmarks extraction is explained. Section III details the fresco construction and its validation. Section V introduces the criteria used to detect the relevant changes in the environment. Results and performances are shown in section VI.

\section{LANDMARKS EXTRACTION}

The problem is to extract the "Opening, Closure, End_of_Closure, Angle_of _Closures" landmarks from the distance data. These landmarks will be used to build the qualitative description of the environment. Four steps are necessary. The first one consists of environment perception (section II-A). It is realised with a laser range finder. The second step describes the construction of the digital representation of the environment (section II-B). It is based on grid representation. The third one develops the technique used for landmarks extraction (section II-C). The fourth step buids the environment representation from landmarks. Distance information is not used in the following sections dealing with frescoes construction, choice of relevant frescoes, and return journey computing to be as close as possible of a human-like perception and description of the environment [22].

\section{A. Perception system: laser range finder}

The size of the non holonomic robot is (width x length) $0.50 \mathrm{~m}$ x $0.75 \mathrm{~m}$. Its linear and angular speeds are up to $1 \mathrm{~m} / \mathrm{s}$ and $2.45 \mathrm{rad} / \mathrm{s}$. Placed at the geometrical centre of the robot with a practical range of $3 \mathrm{~m}$, a panoramic 
telemeter captures the circular environment. It has been decided to consider a $6 \mathrm{~m}$ squared environment to ease the reconstruction process. The robot is always considered as situated at the centre of the environment. Experiments in the following have been made with measurements coming from both a simulated laser range finder and the real telemeter.

This telemeter is able to deliver up to 1024 measurements per rotation with a 5 rpm rotation speed but only 256 information over the 1024 will be used by the fresco construction process. For a regular speed of $1 \mathrm{~m} / \mathrm{s}$, the translation displacement error remains lower than $0.10 \mathrm{~m}$ for one complete rotation of the telemeter. In $100 \mathrm{~ms}$, the rotation of the robot remains lower than $23^{\circ}$.

According to these considerations, we chose to digitise the environment on a $32 \times 32$ cells grid which covers the area seen by the telemeter, each cell representing a $0.1875 \mathrm{~m}$ x $0.1875 \mathrm{~m}$ area. The terms "grid" or "cellular space" will now be considered as equivalent.

The cellular space appears in figure 4a. On the cellular space, a cell will have two states: active or idle. When the laser beam hits an obstacle the corresponding cell will be active and will appear in black in the cellular space. Due to its size the robot can only pass through openings whose width is greater than 3 cells.

\section{B. Construction of the digitised description}

The first step in the symbolic environment consists in generating the digitised environment from the measurements (section II-B.1). The second step performs the segmentation of the cellular space (section II-B.2). Then the third step reorients the cellular space (section II-B.3) to make easier the landmark extraction.

1) Generation of the digitised environment: The method uses evolution laws in the cellular space that act on every cells. For a cell called $C E L L$ the neighbourhood conventions use standard directions according to the Von Neuman neighbourhood. For example, $C E L L_{W}, C E L L_{E}, C E L L_{N}, C E L L_{S}$ are the names of the cells situated westbound, eastbound, northbound, southbound. We add the word Great to name the cells in the second neighbourhood layer (Great West: $C E L L_{G W}$, Great East: $C E L L_{G E}, \ldots$ ). The quadrants are numbered counterclockwise in relation to the lengthwise axis; quadrant 0 is the front right one.

The very first operation consists in the lay-down of the distance measurements onto the grid to create the initial cellular space. This lay-down operation is not a mere polar-to-Cartesian transformation but takes into account the noise introduced in the measurements as they are made while the robot is moving. Noise appears mainly on the form of cells agglomerations (figure 4a). Agglomerations also occur when measurements belong to the border between adjacent cells. Elimination of agglomerations (figures $4 \mathrm{a}$ and $4 \mathrm{~b}$ ) is performed keeping only the cells situated the closest to the robot for obvious safety reasons. The method adopted for this elimination uses evolution laws close to those used in cellular automata.

2) Segmentation of the cellular space: The next operation is the extraction of the segments corresponding to the obstacles from the cellular space. Four directions are considered. In addition to the lengthwise (figure 2a) and crosswise axis (figure 2c), a search for the segments is made onto the two diagonals (figures $2 \mathrm{~d}$ and $2 \mathrm{f}$ ). The extraction laws leave alive a cell owning a neighbour alive in the considered direction. 

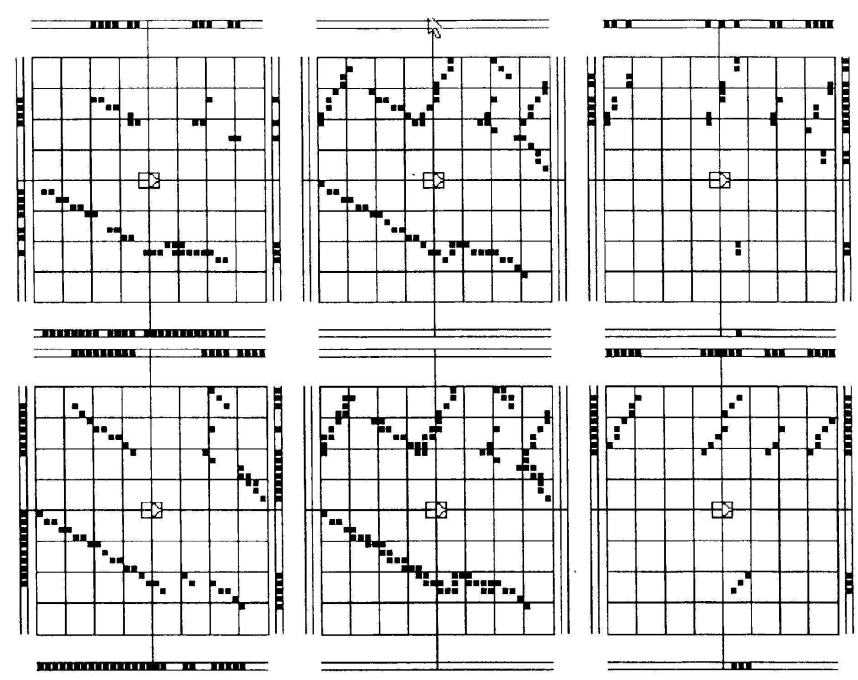

Fig. 2. Extraction of segments in the 4 filtering directions: a(upper left): Lengthwise segmentation, b(upper centre): Refined environment, $\mathrm{c}$ (upper right): Crosswise segmentation, $\mathrm{d}($ lower left): First diagonal segmentation, $\mathrm{e}($ lower centre): Initial measurements, $\mathrm{f}($ lower right): Second diagonal segmentation
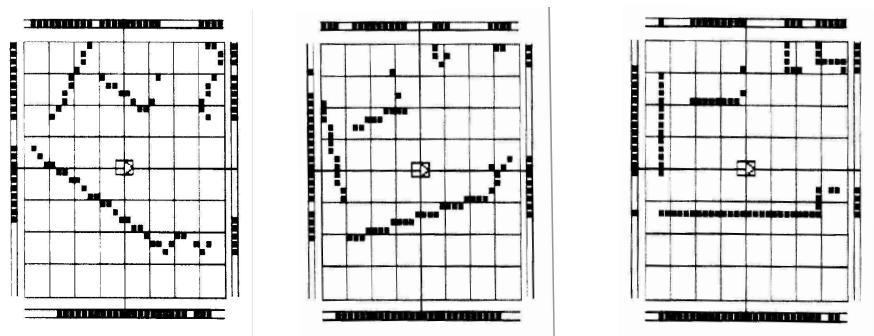

Fig. 3. Example of the effects ot the reorientation of the cellular space: a(left): real world from raw measurements, $\mathrm{b}(\mathrm{centre})$ : $45^{\circ}$ angular shifted cellular space, $\mathrm{c}($ right): reoriented cellular space.

3) Reorientation of the cellular space: As shown in figure 4a, another origin of noise is bound to the oblique walls. These digitised oblique walls take the form of small adjacent segments with junctions without real significance (figure 3a). To eliminate these oblique walls and the noise they introduce we decided to use a second grid on which the measurements are laid with a $45^{\circ}$ angular shift (figure $3 \mathrm{~b}$ ). Superfluous data elimination and segmentation are also applied on this second grid. More details can be found in [23].

\section{Landmarks extraction}

As told in the introduction, environments are described using a fresco made of ordered series of landmarks: "Opening", "Closure" and "Angle_of_Closures". Let us note that an "Angle_of_Closures" must be neighboured by two "End_of_Closure" landmarks. The landmarks extraction first considers the "Opening" elements that are directly extracted from the reoriented cellular space. 
The second operation aims at extracting the "Lenghtwise End_of_Closure" and "Crosswise End_of_Closure" landmarks by evolution laws as well. Figures 4d and 4e examplify the "Angle_of_Closures" and "End_of_Closure” landmarks extraction process. The extracted landmarks are shown positionned on the grids. Finally the set of landmarks constitutes the landmark language shown in table I. Landmarks identity and attributes have been chosen according to the indoor environment in which the robot moves.

\begin{tabular}{|c|c|c|c|c|}
\hline Symbol & Landmark & Position & Off-sight & Certainty \\
\hline ᄂـ & Angle_of_Closure & & & true \\
\hline & End_of_Closure & lengthwise & & true \\
\hline & End_of_Closure & lengthwise & off_sight & false \\
\hline & End_of_Closure & crosswise & & true \\
\hline & End_of_Closure & crosswise & off_sight & false \\
\hline & End_of_Closure & diagonal1 & & false \\
\hline & End_of_Closure & diagonal1 & off_sight & false \\
\hline & End_of_Closure & diagonal2 & & false \\
\hline & End_of_Closure & diagonal2 & off_sight & false \\
\hline & $45^{\circ}$ Angle & lengthwise & & false \\
\hline & $45^{\circ}$ Angle & crosswise & & false \\
\hline & Opening & lengthwise & & true \\
\hline & Breakthrough & lengthwise & & true \\
\hline & Opening & crosswise & & true \\
\hline & Breakthrough & crosswise & & true \\
\hline
\end{tabular}

TABLE I

LANDMARK LANGUAGE USED IN THE FRESCO CONSTRUCTION

With each landmark are associated three qualitative attributes representing three properties. The off-sight attribute is set when the landmark stands on the cellular space border. The position attribute can take the following values: crosswise, diagonal, lengthwise. The certainty attribute is introduced to take into account landmarks that could come from a possible noise introduced in the digitisation process not detected by the previous laws or a still possible bad reorientation. It is false for every landmark (for instance, diagonal "End_of_Closure”, "45o_angles") whose evolution cannot be known.

\section{FRESCO CONSTRUCTION}

In the first phase of the fresco construction, the landmarks are gathered into ordered series of semantic clues. Landmarks are postionned in respect to each others. Each landmark has exactly two neighbours (the last landmark 


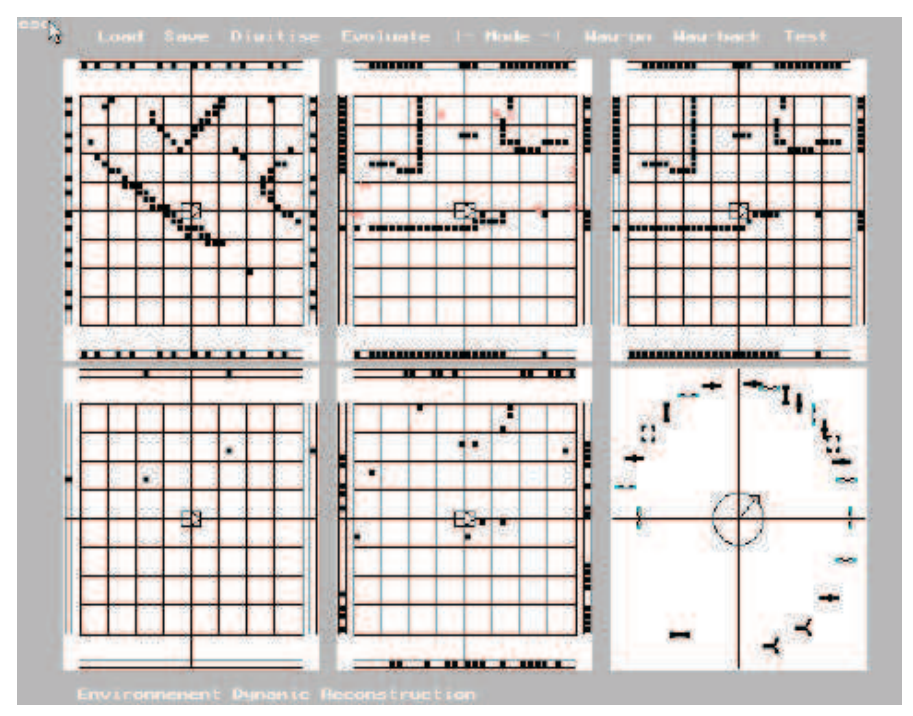

Fig. 4. Example of the digitised constructions: a(upper left): real world from raw measurements, b(upper centre): reoriented cellular space, c(upper right): refined space after superfluous data elimination, d(lower left): Angles_of_Closure extraction, e(lower centre): End_of_Closure extraction, f(lower right): Fresco construction

in the list has the first one as second neighbour). Building the fresco is made using the language presented in table I which gathers landmarks identity and attributes. This operation mainly aims at eliminating the notion of distance to the profit of a spatial series and at highlighting the symbolic representation of the environment.

The second step focuses on the fresco validation. Assuming that there is only one description for one environment, strict laws of neighbourhood are defined. Table II shows these neighbourhood laws that can be interpreted as a set of logical assertions. For example, the neighbours of an Angle_of_Closure can only be Angle_of_Closures or End_of_Closures. For each landmark, the neighbourhood is checked. Every time a fresco is built, the whole set of these rules is applied in order to validate the fresco. If a rule failed, the fresco is not valid.

The validation fails mainly due to a bad landmark extraction process in a very noisy cellular space or a bad reorientation. Making the necessary corrections in the extraction laws to solve these seldom failing cases leads to an increasing of the complexity of the evolution laws, increasing not really justified by the low frequency of the failures.

\section{RESUlTS}

Two kinds of results are given in this section. The first ones deal with fresco construction. The second ones present the behaviour of the robot for a return journey.

Experiments in a realistic environment have been made to validate the reconstruction model and among them, our lab. For various positions along a trajectory in this environment, the frescoes have been built. Fourty five frescoes (Labo1.gif to Labo45.gif) are shown as the robot moves in the lab according to the route drawn (Figure 5). They are built every $0.50 \mathrm{~m}$ for straight forward moves and every $22.5^{\circ}$ for rotations. The number in the names of the 


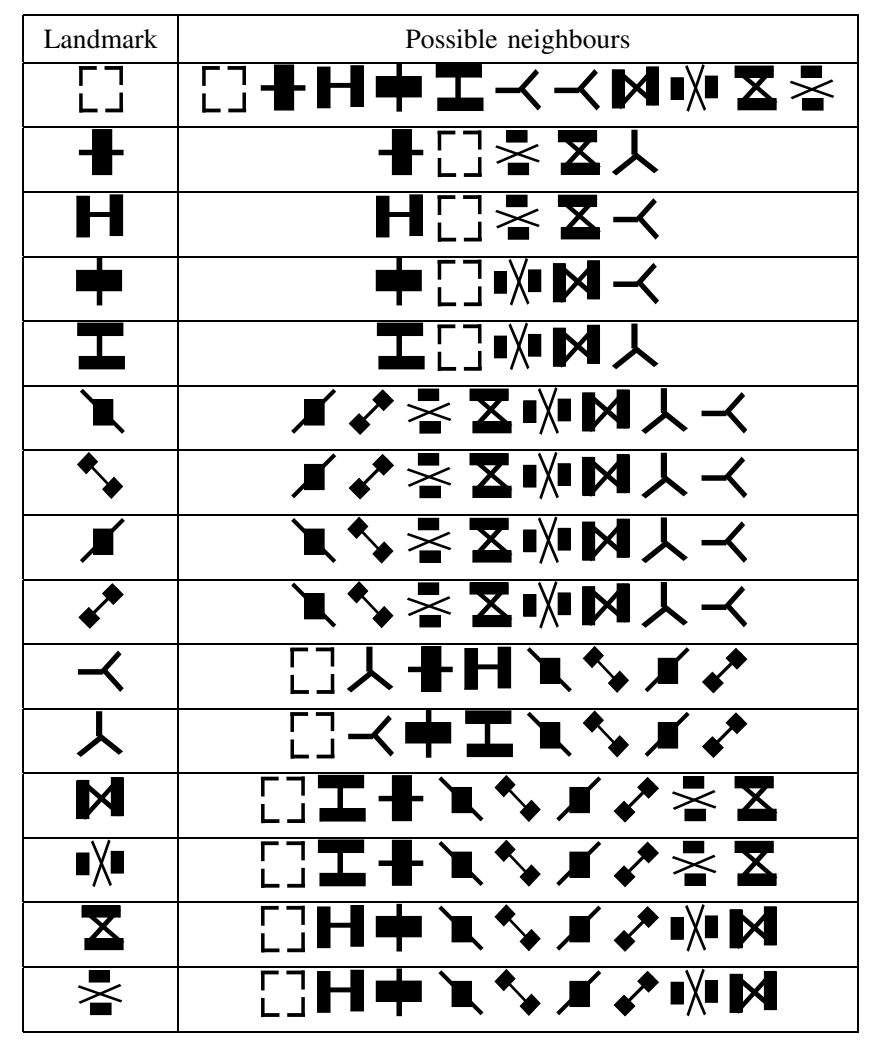

TABLE II

LANDMARKS NEIGHBOURHOOD RULES

gif files corresponds to the robot's position along the route. They represent a large panel of possible situations in indoor environments.

At the very beginning (Figure 6), the robot is situated at the entrance and is well lined up with the wall on its right. No reorientation is needed. The upper middle picture shows the openings the robot could take as well as the orientation to adopt if this opening is chosen. This orientation comes from the computation of the Voronoii graph (not shown) between obstacles [23]. It also can be seen that small openings have been closed in the upper right figure. In figure 7, the upper left cellular space shows the effect of digitisation. Nevertheless, the left side wall is considered as the most continuous one and no reorientation is needed. In Figure 8, the position of the robot shows a case in which the effects of the digitisation are more important. A reorientation is applied. The reorientation angle (in this case, $-45^{\circ}$ ) is represented in the fresco (lower right), the arrow indicates the real orientation of the robot. The active cells in the lower left and lower middle pictures give the localisation of the "Angle_of_Closures" and "End_of_Closures" based on the refined environment in the upper right picture. It is easy see the corresponding relations between, for example, the "Angle_of_Closures" active cells (lower left, quadrants 0 and 4) and the "Angle_of_Closures" symbols in the fresco (lower right). As the route of the robot (Figure 9) is rather straight between positions 10 and 20, no significant reorientation is made and the fresco construction is 


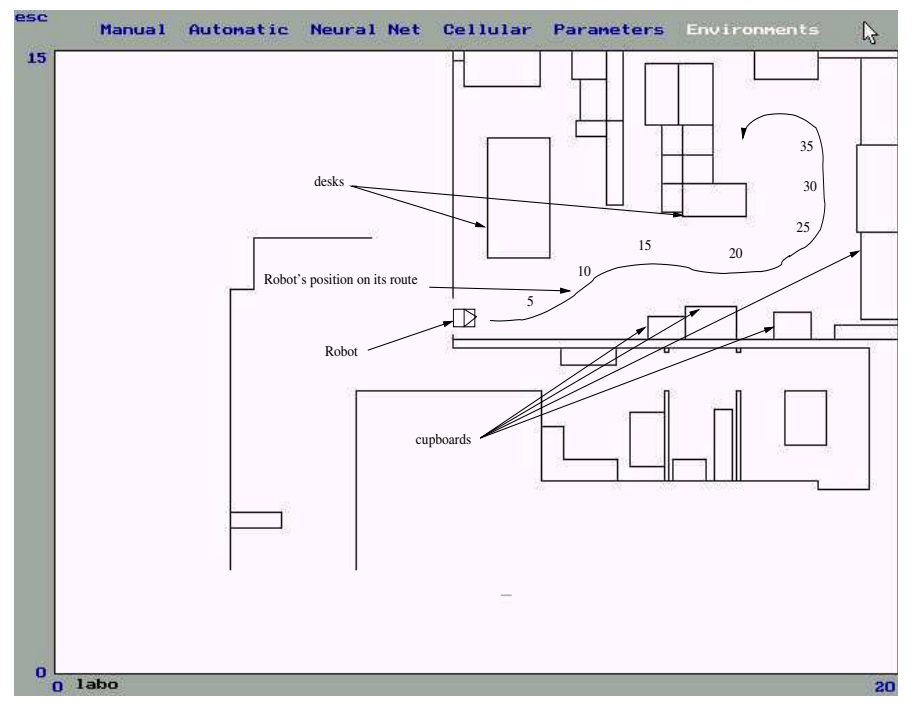

Fig. 5. Test environment: the lab

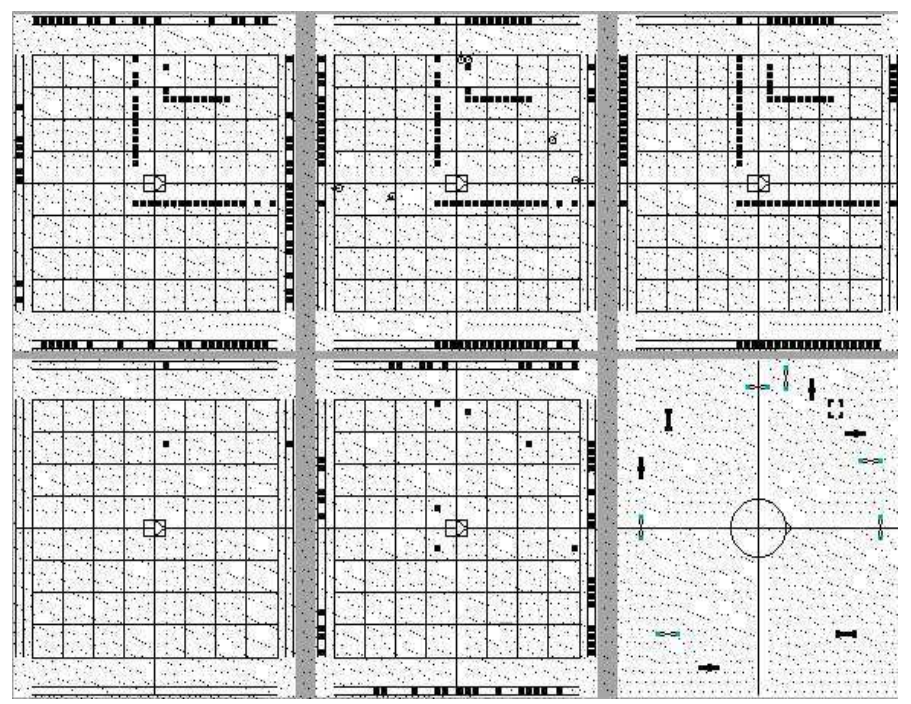

Fig. 6. Test environment: Initial position, position 1 (Labo1.gif)

made without any major difficulties even if the environment includes many breakthroughs and Angle_of_Closures (Figure 10).

The frescos constructed during the trajectory in this realistic environment shows that the described mechanism permits to extract consistent information from the perception system. The consistency of the frescoes ensures they could be used for robot navigation or localisation. A proof of this consistency would be to give to the robot the capacity to execute a return trajectory based only on the use of the frescoes. That means that the frescoes give to the robot a sufficient representation of the environment to allow it to find its way back. That also means that one has to find criteria to detect and keep only the frescoes that bring up relevant changes in the environment. 


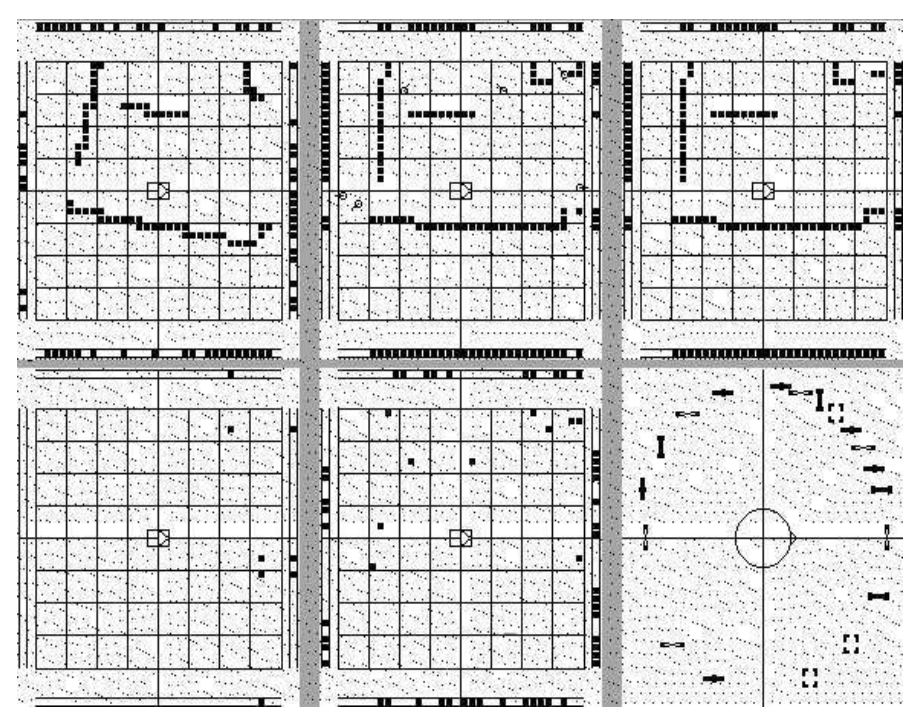

Fig. 7. Test environment: Position 5 (Labo5.gif)

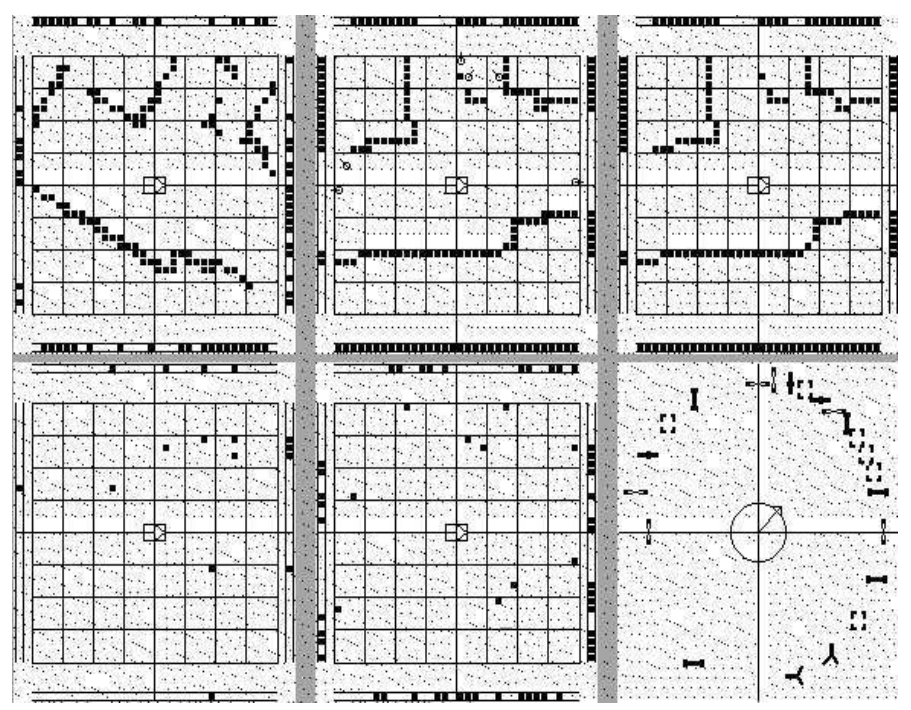

Fig. 8. Test environment: Position 10 (Labo10.gif).

\section{CRITERIA USED TO DETECT RELEVANT CHANGES IN THE ENVIRONMENT EVALUATION}

It is not useful to store all the frescoes that are built [22]. This section presents the first works on two criteria that aims at the selection of the frescoes carrying significant information on the changes in the environment. The first criteria uses a ressemblance function. The second is based on a barycentre displacement evaluation. Finally, the fusion of these criteria and the results of their use in a simulated return journey are given. 

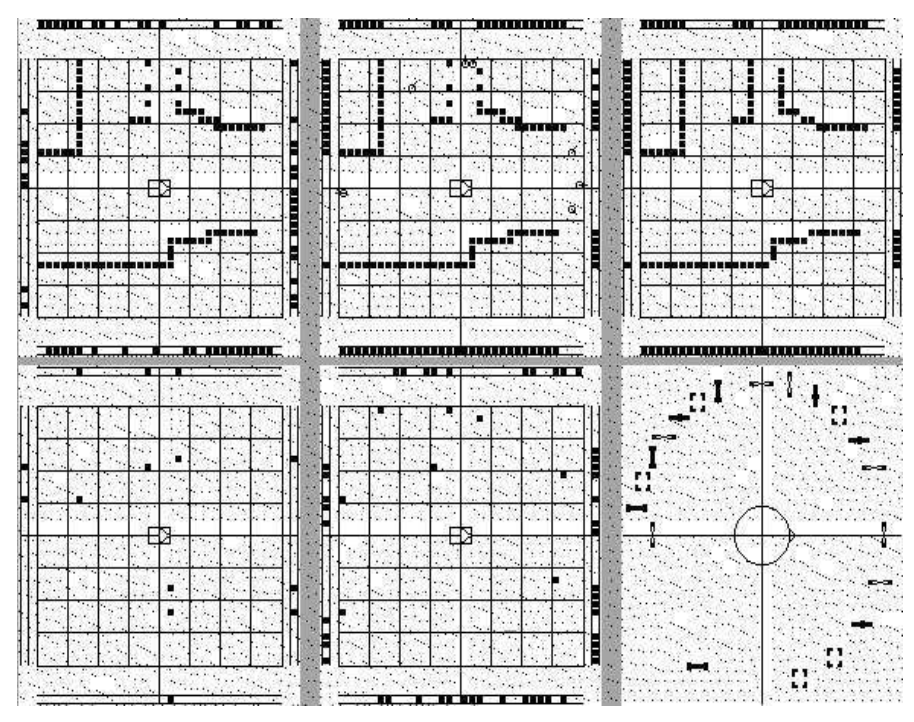

Fig. 9. Test environment: Position 15 (Labo15.gif)
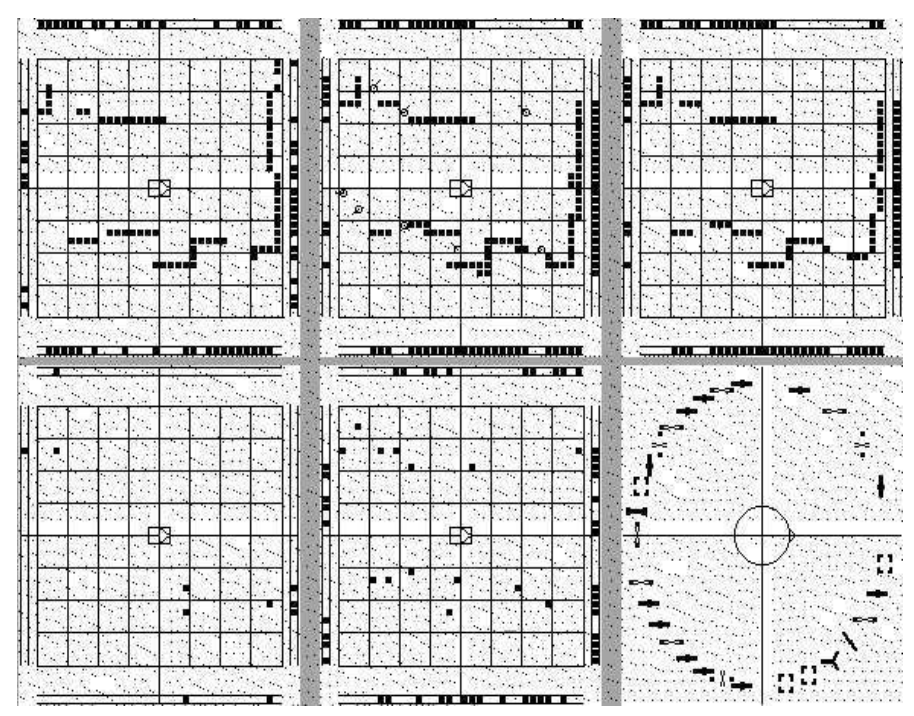

Fig. 10. Test environment: Position 20 (Labo20.gif)

\section{A. Resemblance evaluation between two frescoes}

This criterion uses a nearby principle of that presented in [12]. A correlation function allows to calculate the resemblance between two frescoes. This criterion has been tested in the same environment as that used for the construction and the validation of the frescoes. The use of this criterion shows that the landmarks not defined as certain (section II-C) act as a noise making very difficult the evaluation of the resemblance. From then only were considered the certain elements. The resemblance between two consecutive frescoes is calculated by taking into account the difference between the number of certain landmarks in the corresponding quadrants. The comparison 


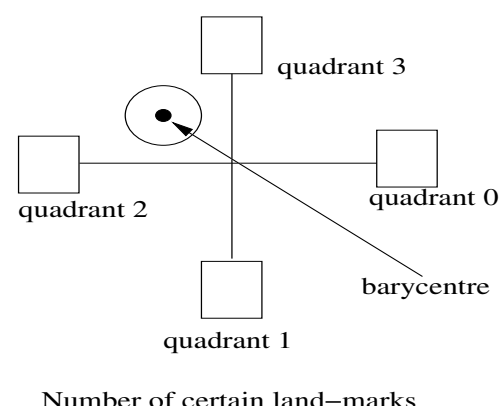

Fig. 11. Barycentre computation between certain landmarks

of this difference with a threshold indicates if the current fresco should be kept or rejected because not bringing enough information

\section{B. Barycentre evaluation between two frescoes}

This criterion is inspired by the works of [24], [25] who measure the distance between two sets(groups). In our case, this notion was very simplified to take into account the real-time constraints in agreement with those embarked on the robot. The number of certain landmarks was positioned as indicated on the figure 11 and the barycentre was positioned. Any variation of the number of elements in a quadran implies a movement of the barycentre. If this displacement is superior to an experimentally adjusted value, the fresco is kept.

\section{Comparison of both criteria}

The experiments carried out in simulated environments are not very satisfactory. For the environments comprising a great number of land-marks, the number of changes in the fresco must be high so that the criterion of resemblance preserves measurement. For simple environments, the number of changes is too weak and not enough frescos is preserved. The use of the criterion of the barycentre in environments for which the number of certain land-marks is weak does not give very good results either, except only for extrememely simple environments. The adaptation of the threshold in the case of the resemblance or the distance between two barycentres to the number of land-marks did not make remarkable improvements either.

\section{Fusion of the agent of classification of environment and the barycentre criteria and resemblance}

The two criteria which have been just described were tested in the various environments. One can however wonder whether one of these criteria is not more powerful according to the type of environment. Previous studies ( [26]) one the uses of on the use of a neural classifier allow the classification of the current into environment 11 classes (Open space, dead-end, T-crossing, X-crossing, ... angle on the left, angle on the right, X_crossing, T_crossing, wall, room, corner, dead end, corridor). We sought to determine if a fusion of the output of this classifier with the criteria of resemblance and barycentre improved the evaluation of the resemblance. A series of tests was carried out 
in these environments. The results of these tests are gathered in the tableau III. For each class of environment we compared the number of frescoes preserved by each criterion. For example, in the case of X-crossing, the criterion of resemblance used to thresholds of 5 and 6 preserve almost all the frescos.

For a threshold of 7 only 5 frescos are preserved. The frescos describing the corridor are:

- before X-crossing,

- during the detection of the opening,

- on arrival in X-crossing,

- at the exit of X-crossing,

- on arrival in the corridor after X-crossing.

These 5 frescos are sufficient even if the fresco corresponding to the position at the centre of the crossing misses.

\section{E. Fusion of the agent of classification of environment and the barycentre and resemblance criteria}

The validation of this work called upon an environment of the interior type of apartment. We simulated the displacement of the robot in this apartment in order to have variable configurations during displacement. The results obtained are much more satisfactory by far. On the one hand, the relationship between the number of memorized frescos and those built are about 1 per 5. This ratio varies in fact according to the speed of the robot. It is more significant (33\%) when speed is $0.50 \mathrm{~m} . \mathrm{s}^{-1}$ that for a speed of $0.25 \mathrm{~m} . \mathrm{s}^{-1}$ for which it is $20 \%$. Indeed, for a significant speed, the differences between two environments are more significant. The speed limits which the robot can reach so that the analysis of resemblance can be carried out was estimated at $1 \mathrm{~m} \cdot \mathrm{s}^{-1}$.

\section{CONCLUSION AND PERPECTIVES}

Human beings, as well as insects [27], use resemblance (or dissimilarity) to compare views of the environment rejecting those that do not bring up new elements without using metrics, only using the occurence of landmarks. In this paper, we present a qualitative method inspired of homing methods [14] to construct the environment surrounding an indoor mobile robot equipped with a $2 \mathrm{D}$ telemetry sensor. To represent the environment the method uses a cellular space on which the distance measurements are laid. The status of the cell represents the existence of an obstacle in the area it is associated with. This method, very robust according to the movements of the robot, has been chosen for its simplicity. Every times distance measurements are made, the cellular space is built. From the cellular space, landmarks are extracted and organised into series called frescoes. Until this point, distance information are not more used. These frescoes, validated according to neighbourhood laws, are stored in the robot's memory and provide minimal information for environment shape understanding. In order to derive the pertinent frescoes that have to be stored along the robot way out, we plan to use a pairing-like method. The first criterion that is primarily being investigated uses a correlation between two frescoes. The landmarks are bounded and a correlation function measures the difference between consecutives frescoes. The second criterion is based on the difference between the barycentre positions of consecutive frescoes [25]. Those frescoes separated by a difference higher than the threshold are considered as pertinent and stored in memory. In both cases the differences are 


\begin{tabular}{|c|c|c|}
\hline Environment & Used criterion & Chosen criterion \\
\hline \multirow{2}{*}{ Left angle } & $\operatorname{bary}(0.75)$ & \\
\hline & resemb(5) & $\sqrt{ }$ \\
\hline \multirow[t]{2}{*}{ Right angle } & bary $(0.75)$ & \\
\hline & $\operatorname{resemb}(5)$ & $\sqrt{ }$ \\
\hline \multirow[t]{6}{*}{$\mathrm{T}_{-}$crossing $\mathrm{n}^{o} 1$} & resemb(4) & \\
\hline & resemb(5) & \\
\hline & resemb(6) & \\
\hline & $\operatorname{resemb}(7)$ & $\sqrt{ }$ \\
\hline & resemb(8) & \\
\hline & $\operatorname{resemb}(6)+\operatorname{bary}(0.75)+5$ & \\
\hline \multirow[t]{2}{*}{$X_{-}$crossing } & $\operatorname{resemb}(5)$ & \\
\hline & resemb(6) & $\sqrt{ }$ \\
\hline \multirow[t]{4}{*}{ Dead end } & resemb $(5)$ & \\
\hline & resemb(4) & \\
\hline & $\operatorname{resemb}(6)+\operatorname{bary}(0.75)+5$ & $\sqrt{ }$ \\
\hline & $\operatorname{resemb}(6)+\operatorname{bary}(0.75)+4$ & \\
\hline Exit/Entrance/Corridor & all & \\
\hline Wall & $\operatorname{resemb}(5)+$ bary $(1)+5$ & $\sqrt{ }$ \\
\hline \multirow[t]{4}{*}{ Room } & resemb $(5)$ & $\sqrt{ }$ \\
\hline & $\operatorname{resemb}(7)$ & \\
\hline & $\operatorname{resemb}(5)+\operatorname{bary}(0.75)+5$ & \\
\hline & $\operatorname{resemb}(5)+$ bary $(1.5)+5$ & \\
\hline \multirow[t]{4}{*}{ Corner } & $\operatorname{resemb}(5)$ & \\
\hline & $\operatorname{resemb}(7)$ & \\
\hline & $\operatorname{resemb}(5)+\operatorname{bary}(0.75)+5$ & \\
\hline & $\operatorname{resemb}(5)+\operatorname{bary}(1)+5$ & $\sqrt{ }$ \\
\hline \multirow[t]{3}{*}{ corridor with zig-zag } & resemb(5) & \\
\hline & $\operatorname{resemb}(7)$ & $\sqrt{ }$ \\
\hline & $\operatorname{resemb}(7)+\operatorname{bary}(0.75)+5$ & \\
\hline
\end{tabular}

TABLE III

CRITERIA RETAINED DURING THE TESTS IN THE ENVIRONMENTS RESULTING FROM THE AGENT OF CLASSIFICATION

with :

resemb(s) : ressemblance criterion with a threshold $=\mathrm{s}$,

bary(s) : barycentre criterion with a threshold $=\mathrm{s}$,

$\operatorname{resemb}\left(s_{1}\right)+\operatorname{bary}\left(s_{2}\right)+s_{3}:$ if the number of certain elements is less than $s_{3}$, the the barycentre criterion is used with a theshold $=s_{1}$, else the resemblance criterion is used with a threshold $=s_{2}$ 
compared with thresholds that are experimentally set up. Nevertheless, the first results in the very changing test environment (Figure 5) are promising.

Remember that the robot has to go in the flat and move back to the handicapped person it is in service of. If it has to return to its starting point, is it able to retrieve its route using only the pertinent frescoes recorded during the way on and those stored during its way back? The robot's return route is not exactly the same than the way on. Therefore, the current fresco does not correspond exactely to the stored frescoes (the $180^{\circ}$ rotation is, obviously, taken into account): the fresco and one situated on the top of the LIFO do not correspond. A first method consists in shifting left or right the current fresco to better fit to one of the stored frescoes [22]. Another method consisting in gathering landmarks into representative sets (alcove, cupboard ...) and using all possible transformations of the current fresco is too bound to human representation and is time consuming. On the contrary, a method grounded on the study of the evolution of very small groups of landmarks seems more promising, simple and low resource consuming. On the other hand, with this method, the robot must anticipate future environments. This anticipation, even if it needs a complete description of all transforms of a fresco, is simpler when the fresco is split into small groups of landmarks. Anticipating frescoes from the current one and comparing them with the stored frescoes seems to be a promising method that allow the robot to choose the right way.

\section{REFERENCES}

[1] G. Wichert, "Selforganizing visual perception for mobile robot navigation," 1996. [Online]. Available: www.rt.e-technik.thdarmstadt.de/g̃eorg/pubs/EUROBOTS96/paper.html

[2] S. A. Allan, "Reconnaissance d'environnement et navigation ractive d'un robot mobile autonome par rseaux de neurones," Ph.D. dissertation, Université d'Evry-Val d'Essonne, feb 1996.

[3] D. Pomerleau, "Neural network perception for mobile robot guidance," Kluwer Academic Publishers, no. 3, pp. 683-699, 1993.

[4] D. Kim and R. Neviata, "A method for recognition and localization of generic objects for indoor navigation," in Proceedings of ARPA Image Understanding Workshop, Monterey, USA, nov 1994, pp. 13-16.

[5] A. Crosnier, "Modélisation géométrique des environnements en robotique mobile," in French Workshop on Robotic Resarch (Journées Nationales de la Recherche en Robotique), Montpellier, France, sep 1999, pp. 83-91.

[6] G. DeSouza and A. C. Kak, "Vision for mobile robot navigation: a survey," IEEE Transaction on pattern analysis and machine intelligence, vol. 24, no. 2, pp. 237-267, feb 2002.

[7] P. Gaussier, C. Joulain, S. Zrehen, and A. Revel, "Image-based homing," in Proceedings of the IEEE International Conference on Intelligent Robots systems, sep 1997, pp. 545-550.

[8] S. Simhon and G. Dudek, "A global topological map formed by local metric maps," in Proceedings of IEEE/RSJ International Conference on Intelligent Robotic Systems, Victoria, B.C., Canada, oct 1998.

[9] S. Oore, G. Hinton, and G. Dudek, “A mobile robot that learns its place," Neural Computation, MIT Press, vol. 9, no. 3, pp. 683-699, 1997.

[10] P. MacKenzie and G. Dudek, "Precise positioning using model-based maps," in Proceedings of IEEE International Conference on Robotics and Automation, San Diego, USA, may 1994, pp. 1615-1621.

[11] M. Franz, B. Schlkopf, and H. Blthoff, "Image-based homing," in Proceedings of the European Conference on Artificial Life, 1997, pp. 236-245.

[12] J. Hong and Al, "Image-based homing," in Proceedings of the IEEE International Conference on Robotics and Automation, Sacramento, USA, apr 1991, pp. 620-625.

[13] S. Judd and T. Collett, "A mobile robot that learns its place," Nature, vol. 392, no. apr, pp. 710-714, 1998.

[14] K. Weber, S. Venkatesh, and S. M. Srinivasan, "Insect inspired robotic homing," Adaptive Behavior, vol. 1, pp. 65-98, 1999. 
[15] M. Tedder and L. E. Hall, “Symbolic processing methods for 3d visual preocessing," 2001. [Online]. Available: www.robotics.uc.edu/papers2001/Maurice2001d.pdf

[16] J. Fleisher, S. Marshland, and J. Shapiro, "Sensory anticipation for autonomous selection of robot landmarks," Foundations, Theories, and Systems, Lecture Notes in Artificial Intelligence, vol. 2684, 2003. [Online]. Available: http://www.cs.man.ac.uk/ fleischj/research.html

[17] J. J. Leonard and H. F. Durrant-Whyte, "Simultaneous map building and localization for an autonomous mobile robot," in Proceedings of the IEEE/RSJ Int. Workshop on Intelligent Robots and Systems IROS '91, New York, NY, USA, May 1991, pp. 1442-1447.

[18] C. M. Smith and J. J. Leonard, "A multiple-hypothesis approach to concurrent mapping and localizastion for autonomous underwater vehicles," in Proceedings of International Conference on Field and Service Robotics, Sydney, Australia,, dec 1997.

[19] B. Kuipers and Y. T. Byan, "A robot exploration and mapping strategy based on a semantic hierarchy of spatial representation," Int. Journal of Autonomous Systems, vol. 8, pp. 47-63, 1991.

[20] H. Choset and K. Nagatani, "Topological simultaneous localization and mapping(slam): Toward exact localization without explicit localization," IEEE Transaction on Robotics and Automation, vol. 17, no. 2, pp. 125-137, 2001.

[21] S. Simhon, "Islands of reliability for dual topological-metric mapping," Ph.D. dissertation, Department of Computer Science,Sep 1998, Centre for Intelligent Machines, McGill University, Montreal, Canada, September 1998.

[22] G. Pradel, S. Avrillon, and L. Garbuio, "Landmark interpretation by means of frescoes in mobile robotics," in Proceedings of the 6th Int. Conf. On Methods and Models in Automation and Robotics, Miedzyzdroye, Poland, aug 2000, pp. 585-592.

[23] F. Bras, G. Pradel, and Z. Jin, "Cellular automata applied to the path generation and environment representation for a mobile robot," in IFAC Motion Control Conference, Munich, Germany, oct 1995, pp. 395-402.

[24] J. Ahuactzin, E. Mazer, and P. Bessiére, "L'algorithme fil d'ariane," Revue d'intelligence artificielle, vol. 9, no. 1, pp. 7-34, 1995.

[25] D. Huttenlocher and Al, "Comparing images using hausdorff distance," IEEE Transactions on pattern analysis and machine intelligence, vol. 15 , no. 9, pp. 850-863, 1993.

[26] G. Pradel and F. Bras, "Qualitative environment description by means of frescoes in mobile robotics," Journal européen des systèmes automatisés, vol. 35, no. 9, pp. 1105-1128, 2001.

[27] T. Collett, E. Dillmann, A. Giger, and R. Wehner, "Visual landmarks and route following in desert ants," Journal of Comparative Physiology A Springler Verlag, vol. 170, pp. 435-442, 1992. 